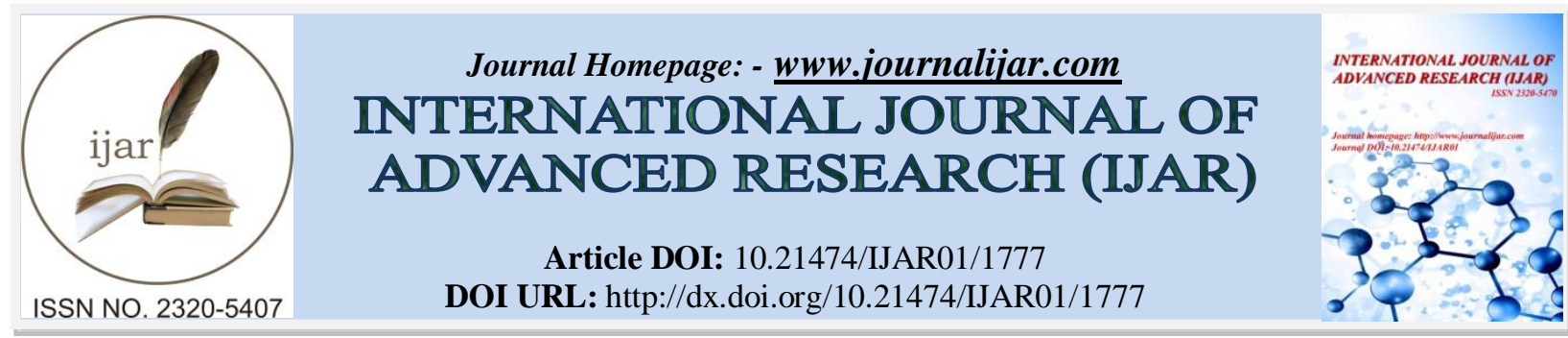

RESEARCH ARTICLE

\title{
METASTASES OSSEUSES ET FRACTURES : ASPECTS EPIDEMIOLOGIQUES ET THERAPEUTIQUES (A PROPOS DE 42 CAS).
}

\author{
J. Boukhris, O. Margad, H. Sallahi, O. Azriouil, M. Daoudi and K. I. Koulali.
}

Service de Traumatologie, Orthopedie, HMA, Marrakech, Maroc.

\section{Manuscript Info}

Manuscript History

Received: 12 August 2016

Final Accepted: 22 September 2016

Published: October 2016

Key words:-

Fracture pathologique, Metastase,

Epidrmiologie, Traitement.

\section{Abstract}

Les métastases osseuses sont les tumeurs les plus fréquentes du squelette. Leur prise en charge reste très discutée et fait l'objet de controverses, elle doit être entreprise au stade pré-fracturaire. Le bilan d'extension de la maladie cancéreuse est important à réaliser avant l'indication thérapeutique. Le traitement chirurgical des ces fractures peut être curatif ou préventif. Ce traitement préventif, qui a l'avantage de se faire à froid, nécessite bien évidemment une étroite collaboration avec les médecins cancérologues, mieux placés pour déceler ces métastases osseuses au stade pré-fracturaire, lors des contrôles de routine. Ainsi les chirurgiens orthopédistes pourront intervenir, sur des malades bien préparés, et par conséquent améliorer la qualité de vie et le confort de ces patients au stade terminal de leur maladie. Notre présent travail a pour objectif d'étudier les aspects épidémiologiques des métastases osseuses à l'origine de fractures pathologiques et d'établir un ensemble de recommandations et de conduite à tenir pour la prise en charge de ces fractures, à la lumière des données récentes de la littérature.

Copy Right, IJAR, 2016,. All rights reserved.

\section{Introduction:-}

Les métastases osseuses sont les tumeurs les plus fréquentes du squelette. Elles se compliquent rarement de fractures. Leur prise en charge reste très discutée et doit être entreprise au stade pré-fracturaire. Le but de notre travail est d'étudier les aspects épidémiologiques de ces métastases osseuses à l'origine de fractures pathologiques, de montrer la supériorité du traitement chirurgical, qu'il soit préventif ou curatif, par rapport aux autres procédés thérapeutiques, et d'établir un ensemble de recommandations et de conduite à tenir pour la prise en charge de ces fractures, à la lumière des données récentes de la littérature.

\section{Materiel d'etude:-}

Notre travail porte sur l'étude rétrospective de 42 cas de fractures métastatiques, colligées au service de Traumatologie-Orthopédie de l'hôpital militaire Avicenne du CHU Marrakech sur une période de dix ans allant janvier 2006 à décembre 2015. Au cours de cette étude ont été précisées les caractéristiques suivantes : l'âge, le sexe, les localisations de ces fractures métastatiques, le bilan radiologique, les modalités thérapeutiques et l'évolution. 
Résultats:-

Les résultats de l'étude de notre série, sont résumés dans les tableaux suivants:

Tableau 1:- La répartition des localisations métastatiques chez les malades de notre série.

\begin{tabular}{|c|c|}
\hline Localisations métastatiques & Pourcentage \\
\hline Fémur & $63 \%$ \\
\hline Humérus & $32 \%$ \\
\hline Tibia & $5 \%$ \\
\hline
\end{tabular}

Tableau 2:- Le pourcentage des tumeurs primitives à l'origine des fractures métastatiques chez les patients de notre série.

\begin{tabular}{|c|c|}
\hline La tumeur primitive & Pourcentage \\
\hline Cancer du sein & $31,5 \%$ \\
\hline Cancer de la prostate & $25 \%$ \\
\hline Cancer du poumon & $23,6 \%$ \\
\hline Cancer du rein & $7 \%$ \\
\hline Les autres tumeurs ostéophytes & $12,9 \%$ \\
\hline
\end{tabular}

Tableau 3 : Les techniques d'ostéosynthèse utilisées chez les patients de notre série.

\begin{tabular}{|c|c|}
\hline Procédés thérapeutiques & Cas \\
\hline Prothèse intermédiaire de hanche & 9 cas \\
\hline Clou gamma & $7 \mathrm{cas}$ \\
\hline EMC du fémur & $15 \mathrm{cas}$ \\
\hline ECM cimenté de l'humérus & $8 \mathrm{cas}$ \\
\hline Lame plaque du fémur distale & $3 \mathrm{cas}$ \\
\hline
\end{tabular}

On a noté un rétablissement de la fonctionnalité des membres avec une reprise d'autonomie, et soulagement des douleurs chez $31 \%$ des cas, et une survie moyenne post fracturaire de 9,5 mois. Les complications post opératoire étaient minimes avec 6 cas de sepsis profond.

\section{Discussion:-}

La prise en charge des fractures pathologiques sur métastases des os long, fait l'objet de controverses, elle doit être entreprise au stade pré-fracturaire. Le traitement chirurgical des fractures métastatiques peut être curatif ou préventif. En effet, selon Duparc, Dossa, Lemaire, l'atteinte corticale sans solution de continuité aboutira inéluctablement à une fracture [1-2-3-4]. Ce traitement préventif, qui a l'avantage de se faire à froid, nécessite bien évidemment une étroite collaboration avec les médecins cancérologues, mieux placés pour déceler ces métastases osseuses au stade pré-fracturaire, lors des contrôles de routine. Ainsi les chirurgiens orthopédistes pourront intervenir, sur des malades bien préparés, et intervenir par conséquent dans l'amélioration de la qualité de vie et le confort de patients au stade terminal de leur maladie.

Qu'elle soit préventive ou réparatrice, une attitude chirurgicale implique le choix entre les différentes techniques d'ostéosynthèse disponibles. Ainsi, nous adoptons:

* Pour les fractures du col fémoral, la prothèse intermédiaire de hanche a été pratiquée 9 fois dans notre série.

* Pour les fractures pertrochanteriennes et trochantero-diaphysaires, l'ostéosynthèse par clou gamma a été réalisé dans 7 cas.

* Pour les fractures diaphysaire du fémur, l'enclouage centromédullaire est la technique la plus utilisée dans notre série (15 cas). Selon Dossa, Geneste, Durandeau et Duparc, La solidité du montage est immédiate et l'utilisation du ciment libère de l'hypothèque de la consolidation ostéogénique [4-8].

* Pour l'extrémité distale du fémur la lame plaque constitue l'indication idéale, préconisé par plusieurs auteurs (Meary) [9-10], dans notre série, la lame plaque a été posée chez 3 de nos patients.

* Pour les fractures de l'humérus, notre préférence est pour l'ostéosynthèse par enclouage centromédullaire cimenté (8 cas). Katzner et Durandeau préfèrent l'enclouage centromédullaire et l'enclouage fasciculé à foyer fermé [5- 6- 8].

Le traitement adjuvant est indispensable dans notre série ainsi que les séries de Lemaire - Katzner-Durandeau. 
La survie moyenne est souvent faible, elle est de 8.5 mois dans notre série. Elle se rapproche de celle rapportée par les auteurs, 9 mois pour Schvingt [5- 6], 8 mois pour Lemaire [7- 9] et 12 mois pour Nayer [2]. Mais cette moyenne de survie est peu significative car le pronostic dépend avant tout du cancer primitif, la vitesse de dissémination métastatique, l'absence de prise en charge des métastases osseuses au stade pré-fracturaire qui pouvait améliorer cette survie et rendait l'acte chirurgical plus aisé.

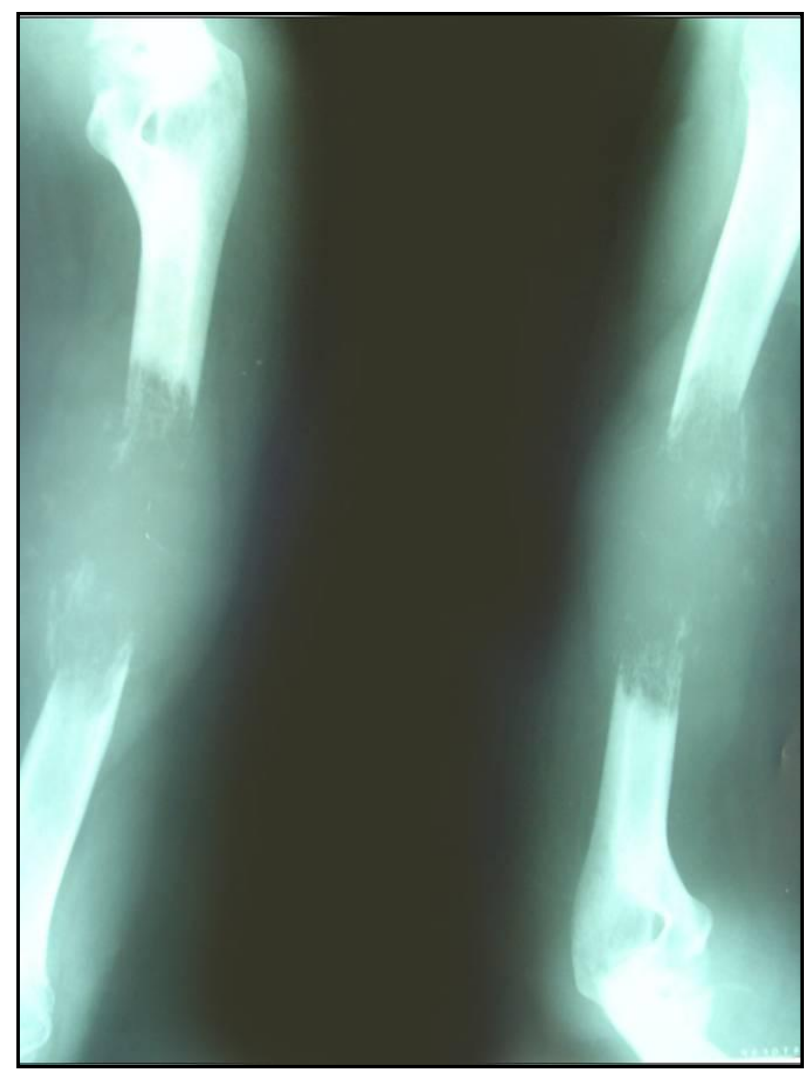

Fig.1:- Métastase ostéolytique de la diaphyse humérale.

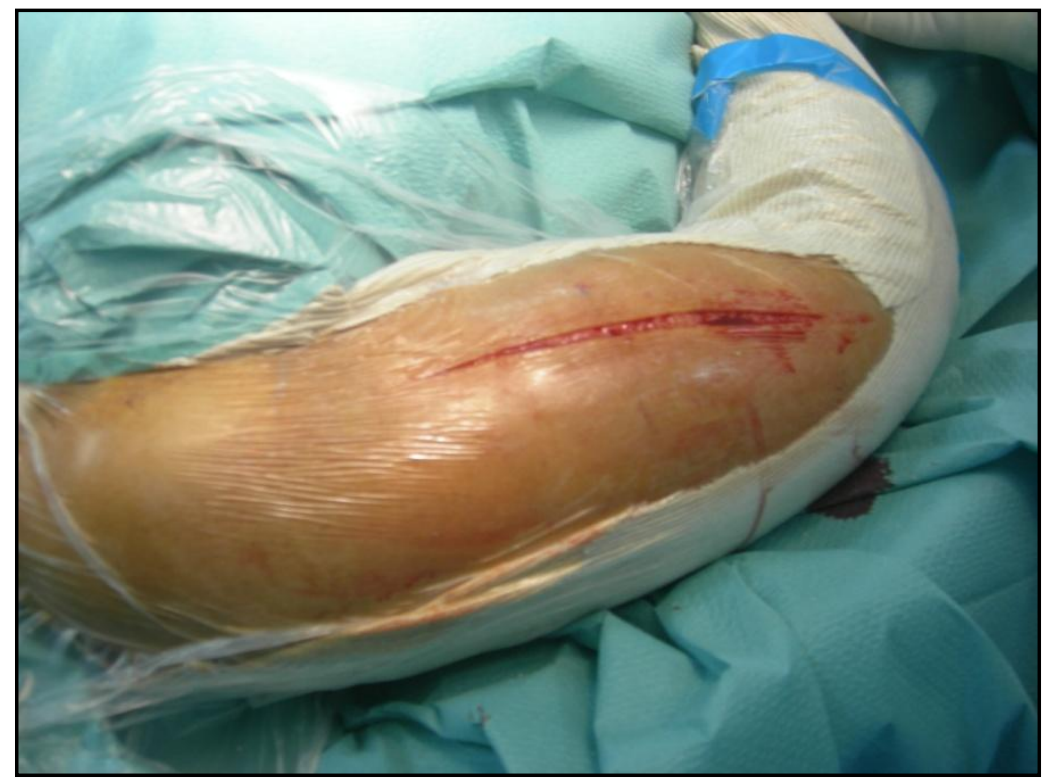

Fig. 2:- Voie d'abord externe. 


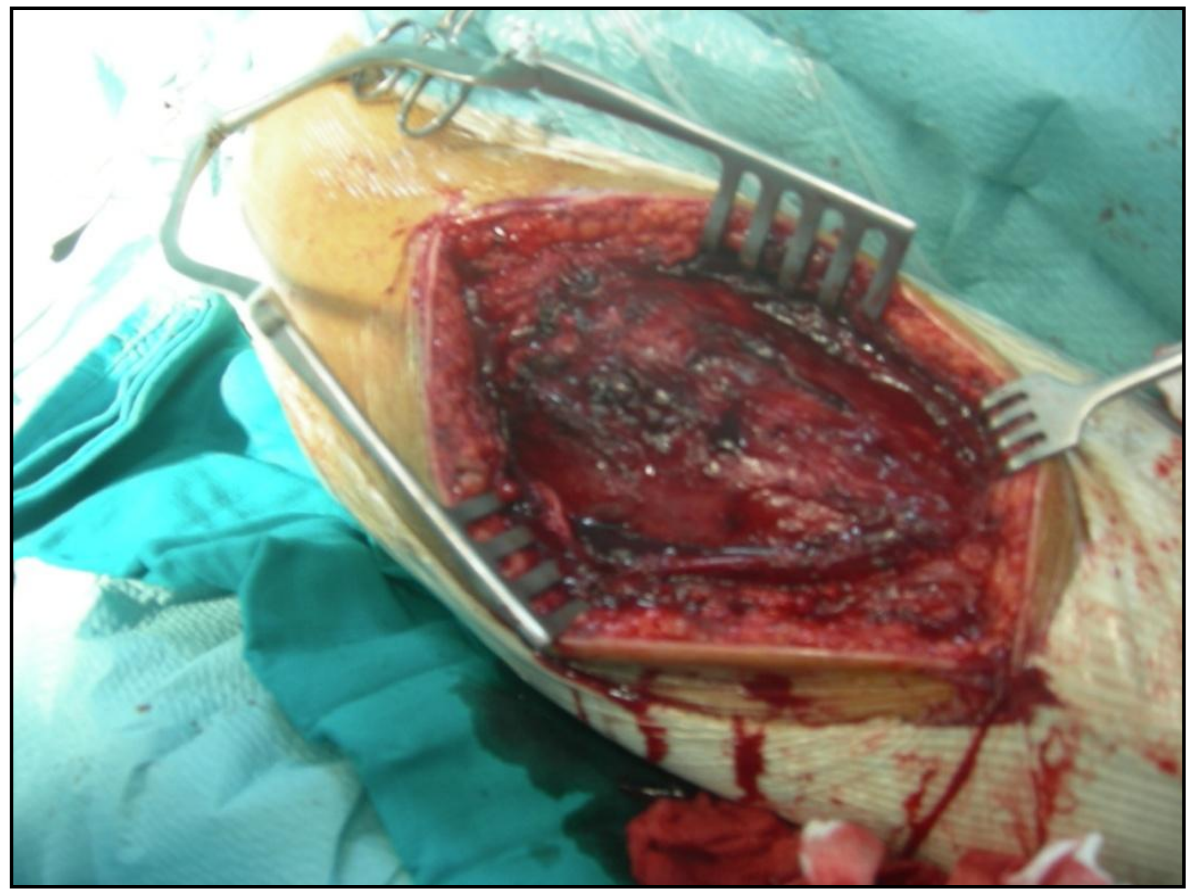

Fig 3:- Aspect macroscopique de la tumeur.

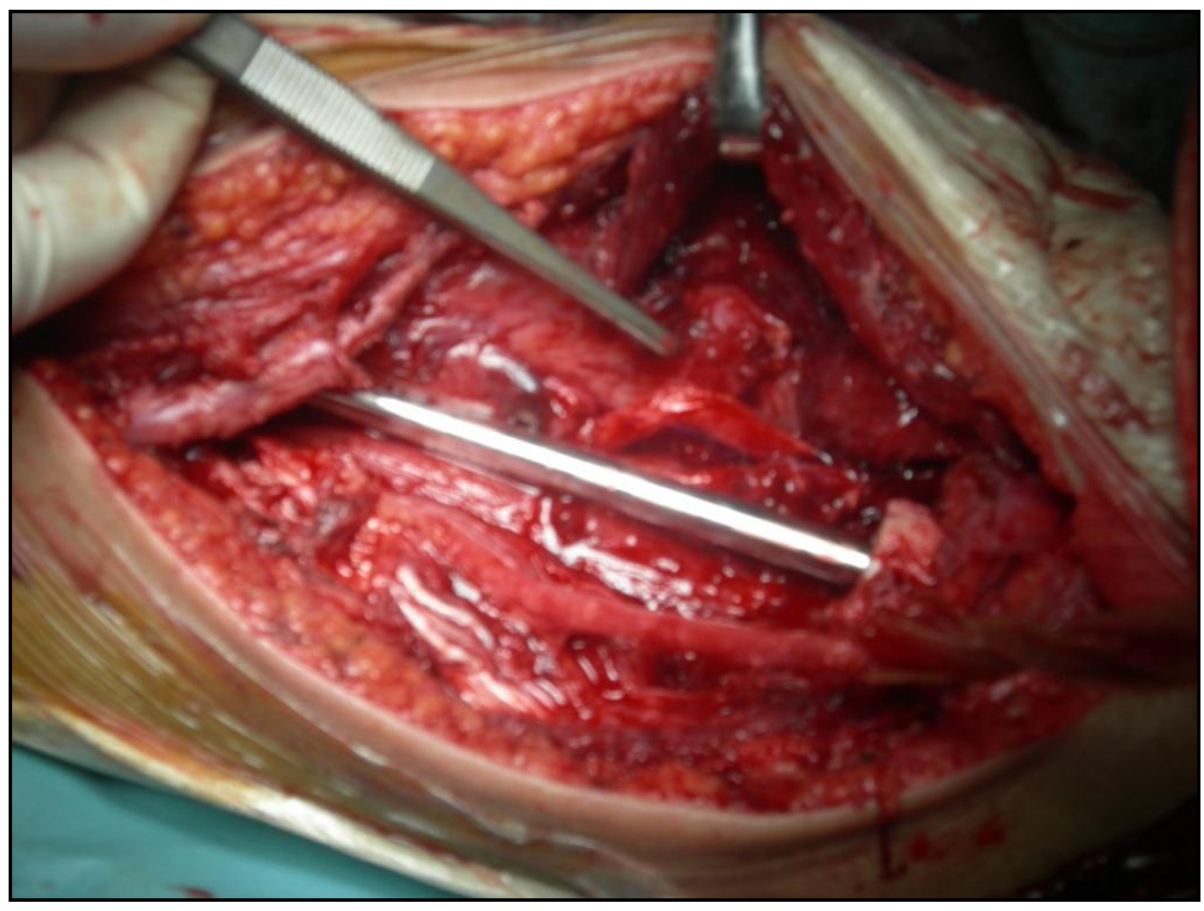

Fig.4:- Résection tumorale et mise en place du clou de l'humérus. 


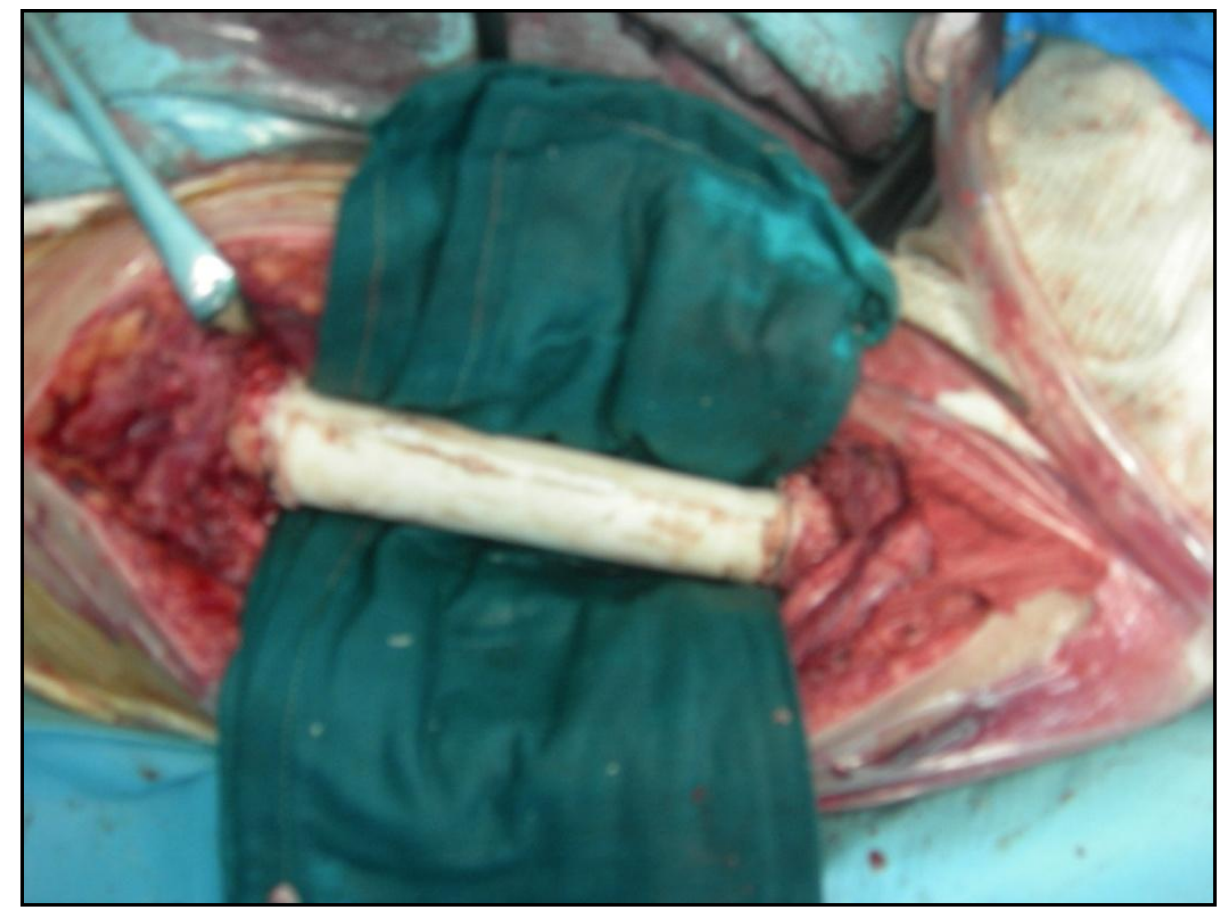

Fig. 5:- Comblement de la perte de substance par un manchon de ciment.

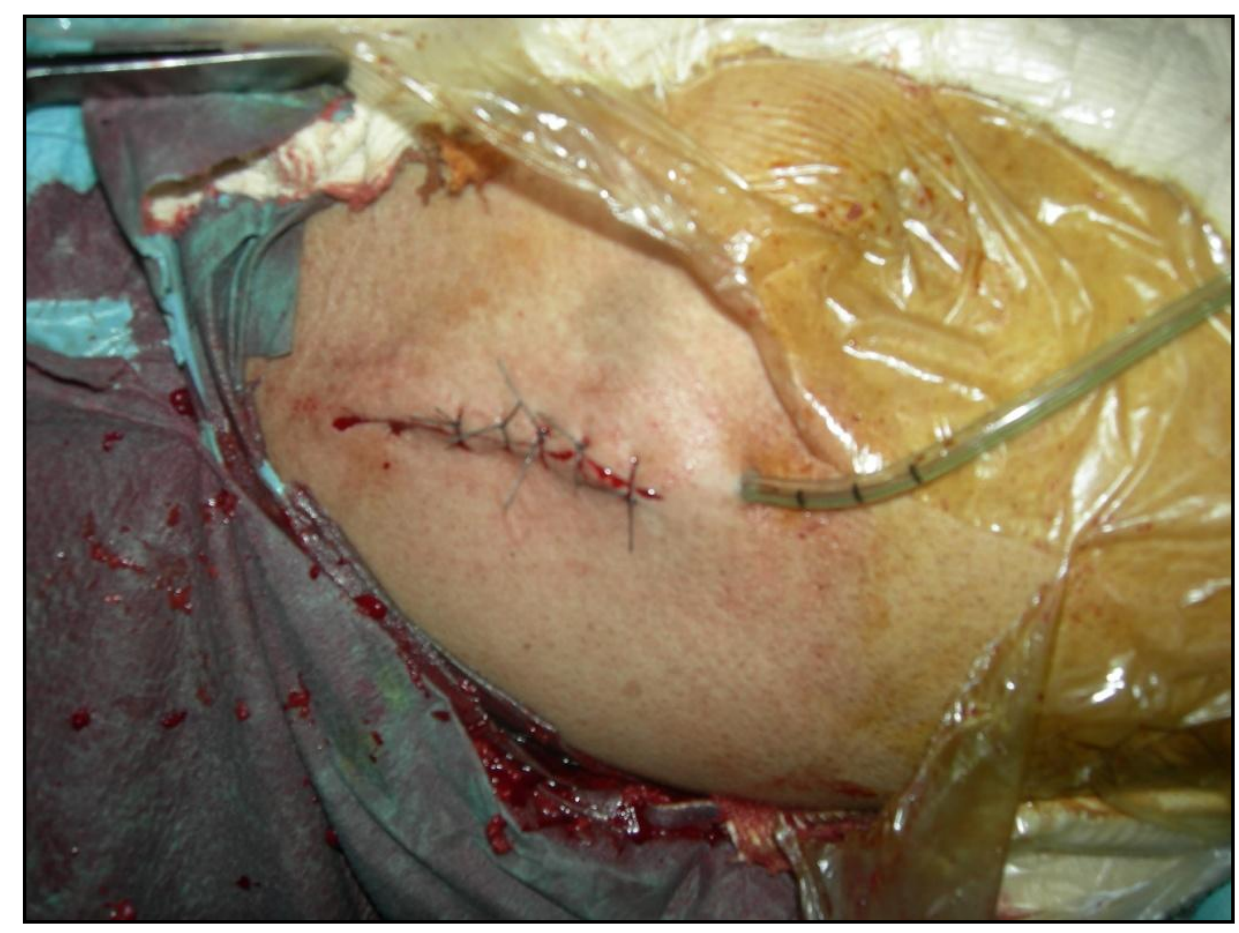

Fig. 6:- Fermeture de la plaie opératoire sur un drain de redon. 


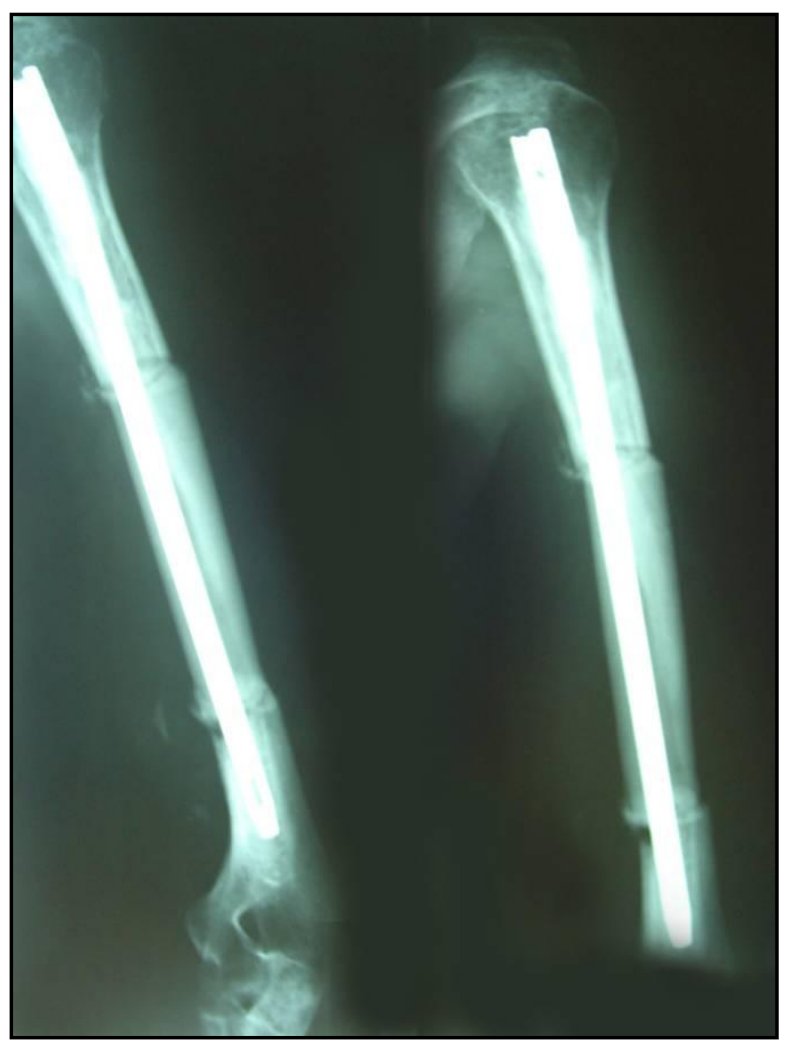

Fig.7:- contrôle radiologique post opératoire.

\section{Conclusion:-}

Les métastases osseuses des os longs doivent être pris en charge au stade pré-fracturaire. Le traitement chirurgical préventif ou curatif des fractures métastatiques des os longs dépasse de loin les autres types de traitement. Son but est d'obtenir l'indolence, la stabilité du foyer de fracture, et la fonction du membre concerné. Ce traitement doit tenir compte de plusieurs facteurs : L'état général du malade ; La localisation de la fracture ; Les types d'ostéosynthèses possibles.

\section{References:-}

1. A.Durandeau et R. Geneste « Traitement chirurgical des fractures métastatiques et des métastases des os longs ». Chirurgie Orthopédique ; N'63; 1977; P 501-517.

2. D.E. Neyer - B. Geulette «Traitement des fractures métastatiques ». Acta Orthopédica Belgica ; Vol 59 ; Sup 1 ; 1993.

3. J.Dossa - Ph.Bemard. « Traitement chirurgical des fractures métastatiques des membres » Montpellier Chirurgie ; N'1 8 ; 1972 ; P 39 - 48.

4. J.Duparc «Le traitement chirurgical des métastases osseuses des membres ». Cahier d'enseignement de SOFCOT; N'18 ; 1983; P 5-30.

5. M. Katzner - E. Schvingt. « Analyse d'une série continue de 500 métastases osseuses traités chirurgicalement ».

6. 6- M.Katzner - E. Schvingt. «L'enclouage selon Ender dans les fractures métastatiques du fémur. » Chirurgie Orthopédique ; N'62 ; 1976 ; P 613-620.Acta Orthopédica Belgica; Vol 59 ; Suppl 1; 1993 ; P 42 - 44.

7. Ph. GIllet - Rlemaire. « Fractures métastatiques et fractures post radiothérapiques . » Acta Orthopédica belgica ; Vol 59 ; Suppl 1 ; 1993 ; P 66-72.

8. R Geneste - A. durandeau. «Traitement chirurgical des fractures pathologiques des membres « Concours Médical 1978 ; 30-9-100-35 ; P 5525-5530.

9. R. Lemaire. « Principes et techniques du traitement chirurgical des métastases osseuses au niveau des os longs ». Aeta orthopédica Belgica- 1993 ; vol 59 ; $\quad$ sup 1 ; P 12-16.

10. R. Meary - M. Postel « Fractures métastatiques du fémur (traitement chirurgical) ». Chirurgie orthopédique; N' 59. 1973; P 91-108. 